\title{
Joint Modelling of CDS and LCDS Spreads with Correlated Default and Prepayment Intensities and with Stochastic Recovery Rate
}

\author{
Péter Dobránszky*
}

This version: November 13, 2008

Original version: August 25, 2008

\begin{abstract}
In this paper, we develop and experiment an intensity based multi-factor model, which incorporates the joint modelling of default, prepayment and recovery risks. In this way, the model provides a link between the credit default swap (CDS) and the loan-only credit default swap (LCDS) markets. The purpose of this paper is to analyse the relationship of the CDS and LCDS markets, and find stochastic risk factors that can explain the joint puzzle of CDS and LCDS spreads. The developed model is simple and analytically tractable, but it is able to capture the most often mentioned characteristics of the markets, namely there is negative correlation between the default and prepayment intensities and also there is positive correlation between the default intensity and the loss given default.
\end{abstract}

Keywords: CDS, credit default swap, LCDS, loan-only credit default swap, pricing, valuation, credit derivatives, credit risk, default risk, prepayment risk, recovery risk, correlated default and prepayment intensities, stochastic recovery rate, continuous time modelling, affine processes, multivariate Ornstein-Uhlenbeck process, Riccati ODE.

*Finalyse SA, Rue de Suisse 18, B-1060 Brussels, Belgium, FORTIS Bank, Risk Management Merchant Banking, Belgium and K.U.Leuven, Department of Mathematics, Belgium. E-mail: peter@dobranszky.com 


\section{Introduction}

Credit markets have seen an explosive growth over the last decade. New products, like CDOs, CPPIs, CPDOs, are brought to the market with an unprecedented complexity. In this paper, we will deal with credit risk derivatives like the credit default swaps (CDSs) and the loan-only credit default swaps (LCDSs). The LCDSs are very similar to the more classical CDSs, but with the extra feature of prepayment built in. Beside the conventional CDSs and their portfolio variant CDOs, also the LCDSs and the portfolios of LCDSs have already found their way into the market. LCDX, for example, is the benchmark index for the loan-only CDSs in North America. In [14], the generic one-factor CDO model is extended with prepayments in order to price tranches of LCDX.

Although many borrowers have both CDS and LCDS issued on, hardly any papers have addressed how to model the CDS and LCDS markets together. Various broker-dealers say that LCDSs should be priced as CDSs disregarding from structural differences, but factoring down according to the difference in the expected recovery rate. As cited in [24] and [4], it means that

$$
\frac{S^{C D S}}{S^{L C D S}}=\frac{1-\bar{R}^{\text {UnsecuredBond }}}{1-\bar{R}^{\text {Loan }}}=\frac{\bar{L}^{\text {UnsecuredBond }}}{\bar{L}^{\text {Loan }}}
$$

should approximately hold. $L$ means here the loss given default. For instance, in case the expected unsecured bond recovery rate is around $40 \%$ and the expected first lien loan recovery rate is at $70 \%$, the fair spread for a CDS should be approximately the double of the LCDS fair spread with corresponding maturity and for the same borrower. Based on the recoveryadjustment formula, one may find a comparison of the CDS-LCDS market figures also in [4].

However, what we see on the market is, that LCDS spreads are sometimes much higher than the recovery-adjusted CDS spreads. This is a puzzle that a model should be able to explain. As it is observed in [23], the 5Y LCDS spreads are usually close to the corresponding recoveryadjusted CDS spreads, but at shorter maturities the LCDS spreads are relatively much higher. According to the authors this indicates that the LCDS and CDS markets are currently rather segmented, though they expect those to move closer together in the future as liquidity increases. However, partly due to the credit crunch, one may conclude that the gap has not decreased, but rather increased recently.

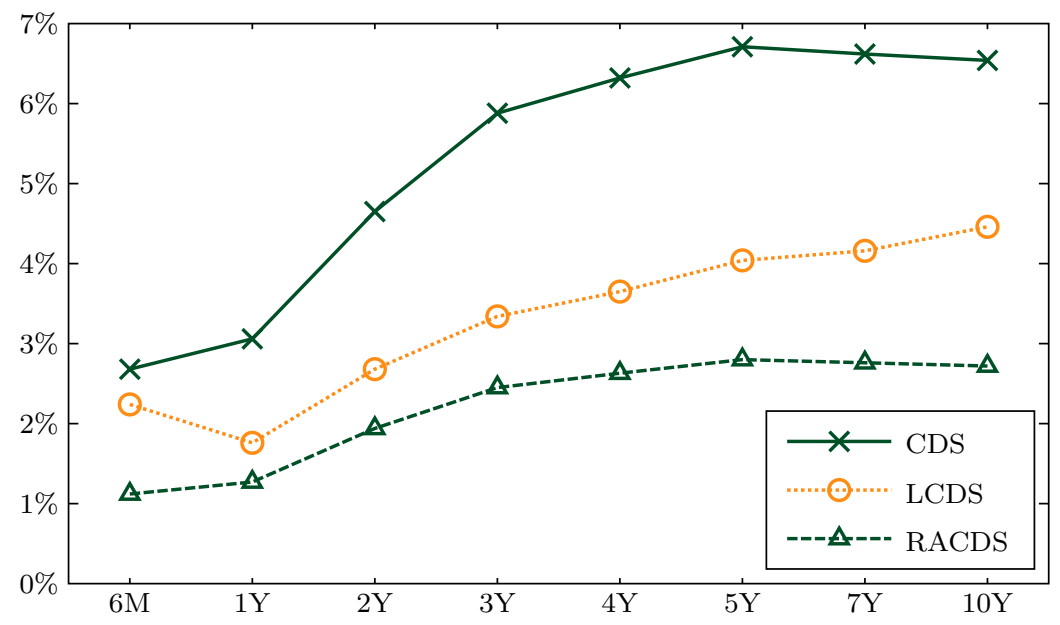

Figure 1: 1st Data Corp CDS (SNRFOR, USD, MR, 40\%) and LCDS (LIEN1, USD, XR, NONCANCEL, $75 \%$ ) curves on 31Jul08 
On Figure 1, we plot the CDS, LCDS and the recovery-adjusted CDS (RACDS) curves for the same borrower. One may observe that the LCDS spreads are much higher than the recoveryadjusted CDS spreads, even though the CDS spreads incorporate additional premium for the restructuring risk. On Figure 2, we show the spread evolution of some liquidly traded CDS and LCDS contracts for the same borrower. Although the LCDS contracts are less liquid than the CDS contracts - this can be seen on the figure as well, - the LCDS and the recovery-adjusted CDS spreads are more or less in line. It is the short 1-year term, where the LCDS was traded permanently above the recovery-adjusted CDS spreads. This corresponds to the observation in [23]. Later, in the following sections, other market examples are given as further puzzles of the CDS and LCDS markets.
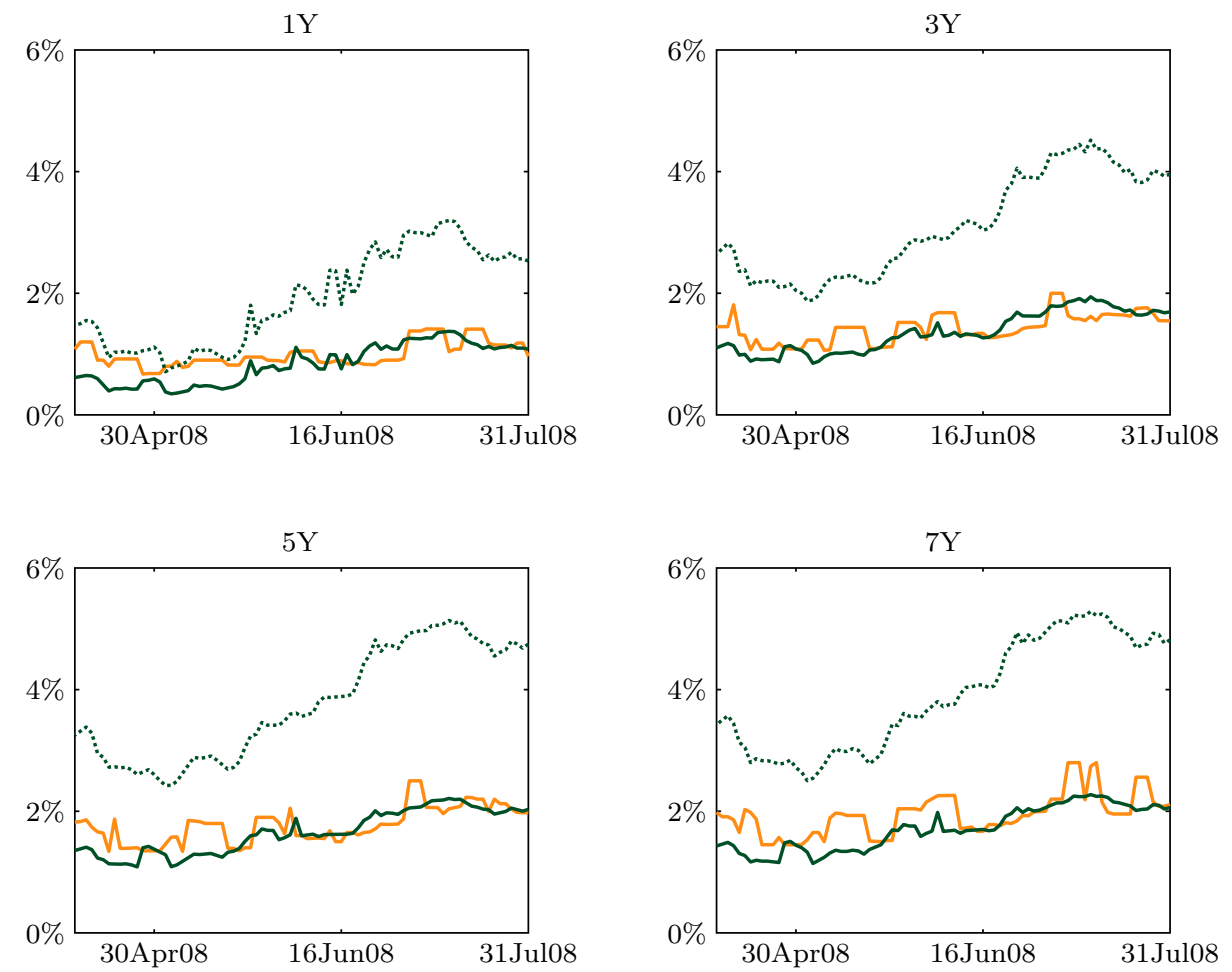

Figure 2: Goodyear Tire \& Rubr Co CDS (SNRFOR, USD, XR, dashed green line), RACDS (green line) and LCDS (LIEN1, USD, XR, NONCANCEL, orange line) spread evolution

Since until recently no stochastic analysis has been seen that would give an explanation for the puzzle of CDS and LCDS spreads, the intention for this paper was to find stochastic risk factors that may provide the link between CDS and LCDS markets and explain the current market figures and their dynamics. With respect to the two most often cited believe that could explain this market puzzle, we build a model and make a stochastic analysis, where we incorporate the possibility of negative correlation between default and prepayment intensities and the possibility of stochastic recovery rate correlated with the default intensity. The model built in this paper is a specialization of the general LCDS model set up in [14].

In this paper, we build our analysis on multivariate affine diffusion processes. In Appendix A, closed-form pricing solutions are derived for a multivariate version of the Gaussian OrnsteinUhlenbeck process, which process is used, for example, also in the Vasicek [26] and Hull-White [21] interest rate models. However, quasi-closed-form solutions can be derived for other affine (jump) diffusion processes as well. Most suitable processes are used also by the quadratic term structure models, for which the reader may be referred to [9]. It is not part of this paper, but 
there are many papers in the literature, which address how to estimate the parameters of such affine processes from historical data.

In this paper, we assume that the risk-neutral dynamics of the driving risk factors are given by the following SDE:

$$
d X_{i}(t)=\kappa_{i}\left(\theta_{i}-X_{i}(t)\right) d t+\sigma_{i} X_{i}^{\beta}(t) d W_{i}^{\mathbb{Q}}(t)
$$

where $\sigma_{i} d W_{i}^{\mathbb{Q}}$ stochastic components are correlated with a given $N \times N$-dimensional covariance matrix $\Sigma$, and $\beta=0$ for the multivariate version of the Gaussian Ornstein-Uhlenbeck process or $\beta=0.5$ for a multivariate extension of the CIR process (named after the well-known CoxIngersoll-Ross term structure model introduced in [13]). For such an extension of the CIR process, known as the Wishart autoregressive process, see [19]. The CIR process gives the variance dynamics of the Heston stochastic volatility model as well, see [20].

This paper is organized as follows: at first we review the similarities and major differences between CDSs and LCDSs in Section 2. Next, in Section 3, we provide a general pricing formula to price CDSs and LCDSs in a common framework. Then, in Section 4, we introduce a model for correlated default and prepayment intensities and we analyse the correlation impact on the LCDS spreads. In Section 5, we extend the model with stochastic recovery rates, while in Section 6 we consider marking-to-market and hedging principles. Finally, we make a summary in Section 7.

\section{Comparison of CDS and LCDS}

A credit default swap is an agreement between two parties to exchange the credit risk of an issuer (reference entity). The buyer of the credit default swap is said to buy protection. The buyer usually pays a periodic fee and profits if the reference entity has a credit event, or the credit worsens while the swap is outstanding. A credit event includes bankruptcy, failing to pay outstanding debt obligations, or in some contracts a restructuring of a bond or a loan. The reference obligation of most corporate credit default swap (CDS) contracts is a Senior Unsecured bond, and credit event usually includes Modified or Modified Modified Restructuring. ${ }^{1}$

The loan-only credit default swap (LCDS) contracts are based on the standard corporate CDS contracts, with several modifications to address the unique nature of the loan market. One difference between CDS and LCDS contracts is the level of the capital structure each contract points to. Namely, the reference obligation of an LCDS contract must be a syndicated secured loan. The loan may be a First, Second or Third Lien note, but it must be secured. With regard to restructuring, LCDS contracts usually exclude restructuring. ${ }^{2}$

Obviously, some part of the CDS spread is the premium related to restructuring, while the bigger part of the spread compensates the expected loss in case of a true default. An interesting study can be found in [7], where the authors compare quotes of CDS contracts with and without restructuring event. They find that the average premium for restructuring risk represents appr. 6-8\% of the swap rate without restructuring. Based on another article, [25], the observed market premium for Modified Restructuring is around 5-10\% of the swap rate without restructuring, though it should be only around 1-6\%. The authors argue, in order to avoid any potential reputational risk still many loan hedgers use CDS contracts with restructuring instead

\footnotetext{
${ }^{1}$ In the US, the Modified Restructuring (MR) is the de facto standard corporate CDS contract, while in Europe the credit event of the standard corporate CDS contract usually includes the Modified Modified (MM) restructuring as well.

${ }^{2}$ In this sense, LCDS contracts follow the convention of CDSs on US high yield issuers, where the credit event includes No Restructuring (XR).
} 
of without restructuring, which causes some overpricing of restructuring, comparing to what historical figures would indicate.

Note, that in case of LCDSs the failure to pay credit events apply to borrowed money, though the deliverables are syndicated secured loans only. This means that a failure to pay on the Senior Unsecured bonds, for example, would trigger a credit event even if the loans were paid as due (assuming they are the same borrower). From modelling point of view this means that after the correction for the difference in restructuring one may assume that the default event of the CDS and the LCDS contracts are driven by the same intensity process (again, assuming the borrower is the same).

In case of default, with regard to the unsecured bonds, the secured loans have first priority on any recovery available to the debt-holders. As a result, the implied recovery rate on loans is much higher than for the bonds, and the risk to principal is therefore much lower for loans. On the market, the assumed average recovery rate for senior unsecured bonds is around $40 \%$, while this rate for secured loans is usually around $70 \%$. The expected loss given default is correspondingly $60 \%$ and $30 \%$. Therefore, we may expect that the LCDS contracts are used to be more exposed to any uncertainty on the recovery rate than the standard CDS contracts.

However, the major difference between CDS and LCDS contracts is that with LCDSs there is a possibility that the loan prepays earlier and hence the instrument is cancellable. Based on [12], $38 \%$ of borrowers that issued leveraged loans exited the senior secured loan market within five years of issuing that loan. Unlike traditional CDSs, LCDS trades are cancellable if no suitable debt remains to deliver upon settlement.

More precisely, the LCDSs in US in most cases do not terminate upon redemption, repayment or other discharge, but are subject to substitution in such circumstances. This substitution process built into US LCDSs ensures that these contracts are not directly cancelled. However, they can be called in whole in case the substitution is unsuccessful. From this point of view, the US LCDSs could be called rather as "semi-cancellable" structures.

Concerning the European LCDSs, those were originally issued with callability feature to terminate or cancel the LCDS contract in the case of redemption, loan repayment or other discharge in full. But, since this callability feature caused many difficulties in unwinding trades, the European market decided to issue non-cancellable - that we call here semi-cancellable LCDSs as well. On the market the semi-cancellable structures are usually more expensive than the cancellable structures, though intuitively we would expect exactly the opposite. On Figure 3 , we plot the puzzle of semi-cancellable and cancellable LCDS curves for the same European borrower and the same underlying loan.

For further details on similarities and differences between credit default swaps and loan-only credit default swaps, see [3] and [5].

\section{Pricing CDS and LCDS}

As mentioned in Section 2, the major difference between CDS and LCDS contracts is, that with LCDSs there is a possibility that the loan prepays earlier and hence the instrument is cancellable. Obviously, both semi-cancellable and cancellable structures contain the risk of prepayment. One may consider a constant variable $a$, which represents the efficiency of substitution as described in Section 2. If $a=1$, there is no substitution, and we say that the structure is fully cancellable. If $a<1$, substitution process applies and the structure is called semi-cancellable.

Disregarding from the difference in the capital structure, which is represented in the recovery rate, one may consider the CDS contracts as LCDS contracts, which are fully non-cancellable, thus the effective prepayment probability is always zero. In this case the theoretical substitution is perfect and $a=0$. In this way, the CDS contracts are migrated into the class of LCDS 


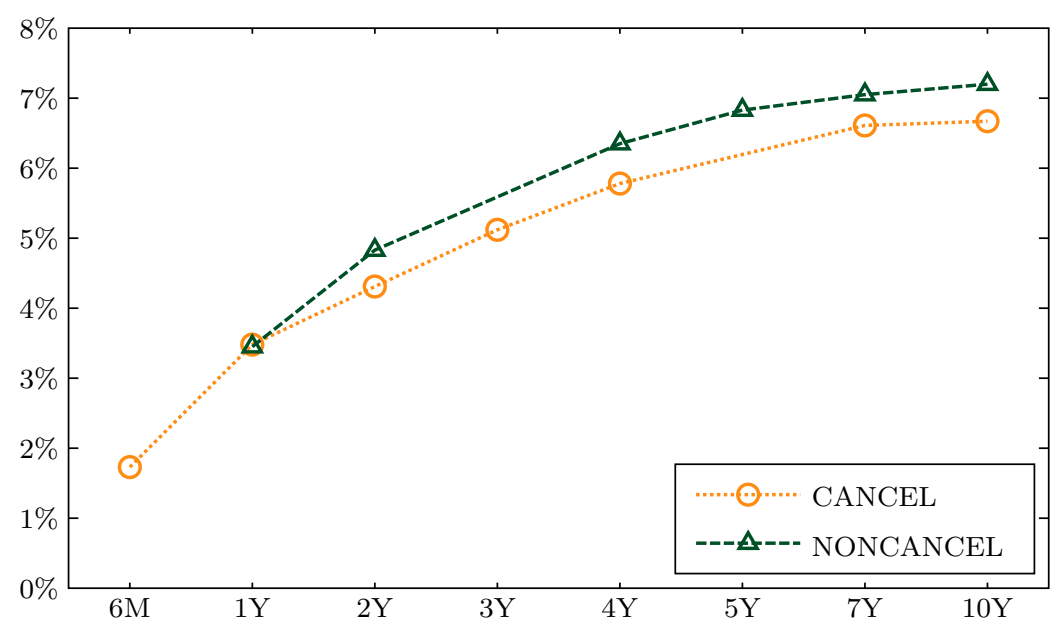

Figure 3: ProSiebenSat 1 Media AG semi-cancellable (NONCANCEL) and cancellable (CANCEL) LCDSs (LIEN1, EUR, MM)

contracts. Therefore, in the followings we will use the notations of [14], in which paper a generic framework for LCDS pricing has already been set up.

During the life of an LCDS contract two kinds of events may be triggered. Either the underlying loan is prepaid without substitution, thus the LCDS is cancelled, or the borrower goes to default. If neither of these two events has been triggered, we say that the LCDS has survived its term, and it was always in the survival state. If during the life of an LCDS contract a cancellation event was triggered first, we say that the LCDS has been cancelled. The LCDS was in the survival state until the cancellation date, and it was in the cancellation state after that. If during the life of the LCDS contract a default event was triggered first, we say that the LCDS has defaulted and the LCDS issuer has to pay the recovery adjusted notional amount to the LCDS buyer. In this case the LCDS was in the survival state until the default time and then it was in the default state.

Let us denote with $T$ now the maturity of the LCDS contract, with $\tau_{c}$ the time when a cancellation event is triggered, with $\tau_{d}$ the time when a default event is triggered, with $P_{\text {cancelled }}(\tau)$ the probability that the contract is cancelled before $\tau$ and with $P_{\text {defaulted }}(\tau)$ the probability that the borrower defaults before $\tau$ and before any cancellation. Obviously, $0 \leq$ $1-P_{\text {cancelled }}(\tau)-P_{\text {defaulted }}(\tau)=P_{\text {survived }}(\tau) \leq 1$ is the probability that the loan will be still on track, i.e. the borrower has not defaulted nor the contract has been cancelled before $\tau$.

$$
\begin{aligned}
P_{\text {cancelled }}(\tau) & =P\left(\tau_{c}<\tau \wedge \tau_{c}<\tau_{d}\right) \\
P_{\text {defaulted }}(\tau) & =P\left(\tau_{d}<\tau \wedge \tau_{d}<\tau_{c}\right) \\
P_{\text {survived }}(\tau) & =P\left(\tau<\tau_{c} \wedge \tau<\tau_{d}\right)
\end{aligned}
$$

It is important to mention that neither $P_{\text {defaulted }}(\tau)$ nor $P_{\text {survived }}(\tau)$ is the same for an LCDS as the corresponding default and survival probabilities of a CDS. Both are conditional on that no cancellation has occurred until $\tau$. Also $P_{\text {cancelled }}(\tau)$ is conditional on that no default has occurred until $\tau$. However, it may happen that the loan is prepaid without substitution before the maturity $\left(\tau_{c}<T\right)$, thus the LCDS is cancelled, but the borrower goes to default after the cancellation, but before the original LCDS maturity $\left(\tau_{c}<\tau_{d}<T\right)$. In this case, the default event is irrelevant for the LCDS contract and we say that the LCDS is in the cancellation state between $\tau_{c}$ and $T$. In the opposite, it is not possible in practice to have a prepayment after a default. 
Clearly, at any given time $\tau$ between 0 and $T$ an LCDS contract is in one of three possible states (defaulted, cancelled, survived). These three states build up to a continuous time Markov chain, where the state at time 0 is "survived", but the stochastic process which represents the state of the LCDS contract may jump at any time to state "defaulted" or "cancelled". Obviously, it is not possible to step out from the "defaulted" neither from the "cancelled" state. However, the intensity, and so the infinitesimal generator of the continuous time Markov chain may be time-dependent as well as stochastic.

Assuming $\lambda_{c}$ to be the intensity that of a cancellation event and $\lambda_{d}$ to be the intensity that of a default event, the infinitesimal generator of the continuous time Markov chain at time $\tau$ may be written as follows.

$$
A(\tau)=\left(\begin{array}{ccc}
-\lambda_{c}(\tau)-\lambda_{d}(\tau) & \lambda_{c}(\tau) & \lambda_{d}(\tau) \\
0 & 0 & 0 \\
0 & 0 & 0
\end{array}\right)
$$

The second and the third lines contain zeros, because it is not allowed to step out neither from the cancelled nor from the defaulted state. In case of an intensity based LCDS model one may model $\lambda_{c}$ and $\lambda_{d}$ as correlated stochastic processes. Nonetheless, also a model with deterministic processes for $\lambda_{c}$ and/or $\lambda_{d}$ fits this framework.

As discussed in Section 2, after the correction for the difference in restructuring, it can be assumed, that the CDS and the LCDS contracts are exposed to the same default event in case the borrower is identical. Therefore, the $\lambda_{d}$ process is identical for CDS and LCDS contracts assuming that the borrower is the same. The intensity that of a cancellation event may be rewritten using the efficiency of substitution as $\lambda_{c}=a \lambda_{p}$, where $\lambda_{p}$ is the intensity that of loan prepayment event. The $\lambda_{p}$ process is identical for CDS and LCDS contracts, and we difference these contracts by $a$. As it is discussed above, $a=0$ for a CDS, $0<a<1$ for a semi-cancellable LCDS and $a=1$ for a cancellable LCDS.

Starting from the infinitesimal generator of the continuous time Markov chain, it is easy to derive the survival probability term-structure $P_{\text {survived }}(\tau)$, while the cancellation and default probability term-structures $P_{\text {cancelled }}(\tau)$ and $P_{\text {defaulted }}(\tau)$ are proportional to each other and sum up to $1-P_{\text {survived }}(\tau)$.

$$
\begin{aligned}
P_{\text {survived }}(\tau) & =E\left[\exp \left(-\int_{0}^{\tau}\left(a \lambda_{p}(u)+\lambda_{d}(u)\right) d u\right)\right] \\
P_{\text {cancelled }}(\tau) & =E\left[\int_{0}^{\tau} a \lambda_{p}(s) \exp \left(-\int_{0}^{s}\left(a \lambda_{p}(u)+\lambda_{d}(u)\right) d u\right) d s\right] \\
P_{\text {defaulted }}(\tau) & =E\left[\int_{0}^{\tau} \lambda_{d}(s) \exp \left(-\int_{0}^{s}\left(a \lambda_{p}(u)+\lambda_{d}(u)\right) d u\right) d s\right]
\end{aligned}
$$

Now, suppose that the LCDS is with a notional $N$ and the spread for protection is denoted with $S$. If the protection fees of the LCDS are payable on the dates $\tau_{1}, \tau_{2}, \ldots, \tau_{n}=T$ (if the LCDS is still on track), which dates are typically quarterly taken, then it results that the present value of the so-called fee leg equals the expected present value of all fees paid.

$$
P V_{\text {Fee }}=S \cdot N \cdot \sum_{i=1}^{n} E\left[D\left(\tau_{i}\right) P_{\text {survived }}\left(\tau_{i}\right) \Delta \tau_{i}+A c c C_{i}\right]
$$


The so-called protection leg, the expected present value of the losses in case of default equals

$$
P V_{\text {Loss }}=N \cdot E\left[\int_{0}^{T}(1-R(s)) D(s) \lambda_{d}(s) \exp \left(-\int_{0}^{s}\left(a \lambda_{p}(u)+\lambda_{d}(u)\right) d u\right) d s\right]
$$

where $R(\tau)$ is the recovery rate corresponding to the capital structure where the contract's underlying points to, $D(\tau)$ denotes the risk-free discount factor from $\tau$ to time zero and $E[$.$] is$ the expectation operator.

The two definitions above are general enough to allow stochastic recovery and interest rates as well, which can correlate with the default and prepayment processes. Furthermore, in line with market conventions, if the default or cancellation happens in between two coupon payment dates, then at the default time accrued coupon $(A c c C)$ and recovery adjusted notional payment are due, or at the cancellation time accrued coupon $(A c c C)$ and the payment of the whole notional are due.

One may recognise, that in the protection leg formula the part of the integrand after $D(s)$ is basically the probability of default differentiated by the time. It is so, because, after some discounting, the present value of the protection leg is the loss given default (LGD) integrated out by the probability of default (PD). As discussed in Section 2, the recovery rates - and so the loss given defaults - for a CDS and for a corresponding LCDS are strongly correlated, but they are different.

\section{Correlated Prepayment Intensity}

It was already suggested in [18], that in case of pricing cancellable LCDS one must take into account not only default probabilities and recovery rates, but also prepayment (refinancing) probabilities and the negative correlation between prepayment and default probabilities. The author argues, the stronger the relationship between default and prepayment rates the higher the LCDS spread. Although in [18] a simplistic model is built up to show also numerically that the stronger the negative correlation the higher the LCDS fair spread, the author takes the very strong assumption that the default probability is not amortized by the prepayment intensity as we formulated our assumptions in Section 3.

We consider this assumption unrealistic. Therefore, we reject the approach of [18], and we start our analysis with correlated intensity processes, where both the default and cancellation probabilities are amortized by each other. Furthermore, as in [18], we assume that the default and the prepayment intensities are negatively correlated with each other. However, in this section, we do not assume yet that there is any other stochastic risk factor like stochastic recovery rate or stochastic discount. The underlying model in this analysis has two stochastic components, $\lambda_{d}$ and $\lambda_{p}$, and both $R(\tau)$ and $D(\tau)$ are supposed to be deterministic.

In such a construction the fee leg can be evaluated relatively easily. The present value of all fees paid is simply the discounted value of the fees, where we discount not only by the money market account and the default intensity, but also by the prepayment intensity. However, the evaluation of the protection leg is much more complicated. Simplifying the pricing problem, one could say that the derivative pays out the loss given default value if the default event is triggered first and pays out nothing if the cancellation event is triggered first.

This problem here is very similar to the problem of pricing first-to-default (FtD) derivatives. One may consider a portfolio of two assets. The first asset is exposed to a default intensity given by $\lambda_{d}$, and the other asset is exposed to a cancellation intensity given by $a \lambda_{p}$. If the payoff of FtD derivative is the loss given default of the first asset in case it is the first asset which goes to 
default first and nothing in case it is the other asset which goes to default first, then the pricing problem is basically identical to the pricing problem of LCDSs. However, as for LCDSs even for FtDs not many researches have been made to price them closed-form. In [15] and [16], the author made some investigations, but at the end he has not derived any solution closed-form.

During the analysis, also the model from [27] was considered. This model uses superposed CIR (squared Bessel) processes. It is assumed that both the default and cancellation intensity processes are linear combinations of independent CIR processes. The correlation between the two intensity processes is introduced by using a common CIR process as follows:

$$
\begin{aligned}
& \lambda_{d}=X+X_{1} \sim \operatorname{CIR}(\kappa, \theta, \sigma)+C I R\left(\kappa_{1}, \theta_{1}, \sigma_{1}\right) \\
& \lambda_{c}=\rho X+X_{2} \sim \rho \operatorname{CIR}(\kappa, \theta, \sigma)+\operatorname{CIR}\left(\kappa_{2}, \theta_{2}, \sigma_{2}\right)
\end{aligned}
$$

where $X, X^{1}$ and $X^{2}$ are three independent CIR processes,

$$
d X_{i}(t)=\kappa_{i}\left(\theta_{i}-X_{i}(t)\right) d t+\sigma_{i} \sqrt{X_{i}(t)} d W_{i}^{\mathbb{Q}}(t),
$$

and $-1<\rho<0$ specifies the negative correlation between $\tau_{d}$ and $\tau_{c}$. The author of [27] argues that taking the parameter restrictions $\kappa=\kappa_{2}, \theta=\theta_{2}$ and $\sigma=\sigma_{2} / \sqrt{|\rho|}$, the positivity of the cancellation intensity process is assured and the equations simplify to

$$
\begin{aligned}
& \lambda_{d}=X+X_{1} \sim \operatorname{CIR}\left(\kappa_{2}, \theta_{2}, \sigma_{2} / \sqrt{|\rho|}\right)+\operatorname{CIR}\left(\kappa_{1}, \theta_{1}, \sigma_{1}\right) \\
& \lambda_{c}=\rho X+X_{2} \sim \operatorname{CIR}\left(\kappa_{2},(\rho+1) \theta_{2}, \sigma_{2}\right)
\end{aligned}
$$

However, strictly speaking $\rho$ is not a correlation parameter. The $\rho$ as parameter has impact on the univariate $\lambda_{d}$ and $\lambda_{c}$ processes as well. This means, for instance, that $\rho$ has impact on the CDS spreads as well, though it should not, because the CDS spreads are based only on the default intensity process, and not on the cancellation intensity process, neither on their correlation.

In [27], the impact of $\rho$ on the LCDS spreads is analysed, and the author concludes that "increasing the (negative) correlation between default and loan cancellation increases the LCDS spread". However, this conclusion is wrong. By changing $\rho$ the LCDS spreads changed in [27] mainly, because the parameter $\rho$ has a direct impact on the volatility of the default intensity, thus also on the default probabilities and so on the spreads. This impact should not be confused with the impact of the statistical correlation between the intensity processes.

In this paper, we intend to analyse the real contribution of the default-prepayment intensity correlation to the LCDS spreads. Unfortunately, the model in [27] does not allow to carry out such an analysis. Therefore, we had to build a model, where it is possible to freely choose the parameters of the stochastic risk factors. There should be one parameter which represents the statistical correlation between the intensity processes, and this parameter should not have any impact on the univariate intensity processes.

For this purpose, we choose a multivariate extension of the Gaussian Ornstein-Uhlenbeck process as introduced in Section 1. In this model, the dynamics of the intensity processes are given by

$$
\begin{aligned}
d \lambda_{d}(t) & =\kappa_{d}\left(\theta_{d}-\lambda_{d}(t)\right) d t+\sigma_{d} d W_{d}^{\mathbb{Q}}(t) \\
d \lambda_{p}(t) & =\kappa_{p}\left(\theta_{p}-\lambda_{p}(t)\right) d t+\sigma_{p} d W_{p}^{\mathbb{Q}}(t)
\end{aligned}
$$

where $\operatorname{Corr}\left(d W_{d}^{\mathbb{Q}}, d W_{p}^{\mathbb{Q}}\right)=\rho$. This $\rho$ parameter has impact only on the joint dynamics and not on the univariate intensity processes. It is true that this model allows negative intensities, 
but the probability that the intensity would go below zero can be neglected if the parameters are chosen properly. This restriction is already well-known from the Vasicek and Hull-White interest rate models. Nonetheless, even in case of CIR processes, the Feller condition has to be fulfilled in order to avoid negative intensities.

Using the results from Appendix A, the survival, default and cancellation probabilities defined by this reduced form model can be derived closed-form:

$$
\begin{aligned}
P_{\text {survived }}(\tau) & =\mathcal{I}_{0, \tau}^{\mathbf{X}}\left(\left[\begin{array}{ll}
a & 1
\end{array}\right]^{T},\left[\begin{array}{ll}
0 & 0
\end{array}\right]^{T}\right) \\
P_{\text {cancelled }}(\tau) & =\int_{0}^{\tau} \mathcal{L}_{0, s}^{\mathbf{X}}\left(\left[\begin{array}{ll}
a & 1
\end{array}\right]^{T},\left[\begin{array}{ll}
0 & 0
\end{array}\right]^{T},\left[\begin{array}{ll}
a & 0
\end{array}\right]^{T}\right) d s \\
P_{\text {defaulted }}(\tau) & =\int_{0}^{\tau} \mathcal{L}_{0, s}^{\mathbf{X}}\left(\left[\begin{array}{ll}
a & 1
\end{array}\right]^{T},\left[\begin{array}{ll}
0 & 0
\end{array}\right]^{T},\left[\begin{array}{ll}
0 & 1
\end{array}\right]^{T}\right) d s
\end{aligned}
$$

where $\mathbf{X}=\left[\begin{array}{ll}\lambda_{p} & \lambda_{d}\end{array}\right]^{T}$. Obviously, the survival and default probabilities for a non-cancellable CDS can be obtained either by setting $a=0$ or by simplifying the above equations using only a univariate Gaussian Ornstein-Uhlenbeck process.

Intuitively, one may argue that increasing the (negative) correlation between the intensity processes the variance of $\left(\lambda_{d}(t)+\lambda_{p}(t)\right)$ is decreasing. Therefore, the survival probabilities and the present value of the fee leg decrease, while the default probabilities and the present value of the protection leg increase. This implies that following our model assumptions intuitively the higher the (negative) correlation the higher the fair LCDS spread.

In order to test this intuition and so the impact of the correlation we set up hypothetical scenarios. Both for the default and prepayment processes we choose the same $\kappa, \theta$ and $\sigma$ parameters. We set the CDS recovery rate to $40 \%$ and the LCDS recovery rate to $70 \%$. We test the relationship of the CDS and LCDS spread curves without correlation $(\rho=0)$ and with a strong negative correlation $(\rho=-0.9)$. We calculate also the recovery-adjusted CDS spreads (RACDS) as defined in Section 1. The results, which are calculated for different initial process states $X_{0}$, are shown in Table 1.

\begin{tabular}{c|l|ccccccc}
\multicolumn{2}{c|}{$\left(\kappa_{i}, \theta_{i}, \sigma_{i}\right)=(0.5,6 \%, 2 \%)$} & $6 \mathrm{M}$ & $1 \mathrm{Y}$ & $2 \mathrm{Y}$ & $3 \mathrm{Y}$ & $4 \mathrm{Y}$ & $5 \mathrm{Y}$ & $7 \mathrm{Y}$ \\
\hline$X_{i}(0)=3 \%$ & CDS & $2.04 \%$ & $2.21 \%$ & $2.45 \%$ & $2.63 \%$ & $2.76 \%$ & $2.68 \%$ & $2.99 \%$ \\
& RACDS & $1.02 \%$ & $1.10 \%$ & $1.22 \%$ & $1.31 \%$ & $1.38 \%$ & $1.43 \%$ & $1.49 \%$ \\
& LCDS $(\rho=0)$ & $1.02 \%$ & $1.10 \%$ & $1.22 \%$ & $1.31 \%$ & $1.37 \%$ & $1.41 \%$ & $1.47 \%$ \\
& LCDS $(\rho=-0.9)$ & $1.02 \%$ & $1.10 \%$ & $1.23 \%$ & $1.31 \%$ & $1.38 \%$ & $1.42 \%$ & $1.48 \%$ \\
\hline$X_{i}(0)=6 \%$ & CDS & $3.59 \%$ & $3.58 \%$ & $3.57 \%$ & $3.56 \%$ & $3.56 \%$ & $3.55 \%$ & $3.55 \%$ \\
& RACDS & $1.79 \%$ & $1.78 \%$ & $1.78 \%$ & $1.78 \%$ & $1.77 \%$ & $1.77 \%$ & $1.77 \%$ \\
& LCDS $(\rho=0)$ & $1.79 \%$ & $1.79 \%$ & $1.78 \%$ & $1.78 \%$ & $1.78 \%$ & $1.78 \%$ & $1.77 \%$ \\
& LCDS $(\rho=-0.9)$ & $1.79 \%$ & $1.79 \%$ & $1.79 \%$ & $1.79 \%$ & $1.79 \%$ & $1.79 \%$ & $1.79 \%$ \\
\hline$X_{i}(0)=9 \%$ & CDS & $5.13 \%$ & $4.95 \%$ & $4.69 \%$ & $4.50 \%$ & $4.36 \%$ & $4.26 \%$ & $4.12 \%$ \\
& RACDS & $2.56 \%$ & $2.47 \%$ & $2.34 \%$ & $2.25 \%$ & $2.18 \%$ & $2.13 \%$ & $2.06 \%$ \\
& LCDS $(\rho=0)$ & $2.57 \%$ & $2.48 \%$ & $2.35 \%$ & $2.27 \%$ & $2.20 \%$ & $2.15 \%$ & $2.09 \%$ \\
& LCDS $(\rho=-0.9)$ & $2.57 \%$ & $2.48 \%$ & $2.36 \%$ & $2.27 \%$ & $2.21 \%$ & $2.16 \%$ & $2.10 \%$ \\
\hline
\end{tabular}

Table 1: CDS and LCDS Curves with Correlated Default and Prepayment Intensities

After several tests we may conclude that the possibility of prepayment usually flatten the spread curve. If the CDS curve is already flat, the cancellation probability and the correlation 
between the default and prepayment intensity processes have basically no impact on the LCDS curve. However, if the CDS curve is contango, as it is usually like that on the market, due to the flattening effect the LCDS spreads are slightly below the recovery-adjusted CDS spreads. This result is in line with the puzzle shown on Figure 3, where the cancellable LCDS curve is slightly below the non-cancellable LCDS curve.

Concerning the correlation impact on the LCDS curve, as expected, increasing the (negative) correlation between the default and prepayment intensities, ceteris paribus, the LCDS spreads increase. However, as we may found the impact of the correlation on the LCDS spreads is insignificant. The correlation between the default and prepayment intensity processes does not explain the general market puzzle of CDS and LCDS spreads. Therefore, in the next section we incorporate further stochastic factors into our model.

\section{Correlated Recovery Rate}

It has already been concluded in several researches, like in [1], that the stochastic behaviour of the recovery rate and its negative correlation with the default intensity are important ingredients of modelling credit derivatives. However, up to now very few papers have been published which address the problem of pricing credit derivatives based on stochastic recovery rate. [2] was maybe the first paper that addressed the decomposition of default and recovery risks. Unfortunately, the proposed model suffers from the fact that there is only one factor, the interest rate, to explain both interest rate, default, and recovery risks.

In [16], the author set up a theoretical framework and proposes to use affine processes for modelling stochastic recovery rate, but he does not make any application. In [10] and [11], the author develops a multi-factor CDS pricing model with recovery risk. However, he models the stochastic recovery rate as weighted sum of independent CIR and Gaussian Ornstein-Uhlenbeck processes. This construction has the problem, that due to the number of parameters the model is not tractable anymore. It is hardly feasible to separate the effect of the different risk factors. Moreover, the recovery rate can easily go under $0 \%$ or above $100 \%$. In [22], the author sets up the basics for modelling stochastic recovery rates in reduced-form models, but he provides tractable solution only for a discrete-time model.

The intention here is to build up a continuous-time stochastic recovery model, which is as simple as possible and fits into our modelling framework introduced in Section 3. As in [8], we assume that the recovery payment is a fraction of the face value.

$$
R(s)=1-L(s)
$$

where $\{L(t), s>t\}$ is an adapted process bounded by 0 and 1 and represents the loss given default. We will include the stochastic recovery rate as risk factor into the model through $\delta$, which will denote here the natural logarithm of the loss given default.

$$
L(s)=\exp (\delta(s))
$$

We assume that $\delta$ is a component of our affine state vector. Furthermore, we assume that the recovery rate is stochastic and negatively correlated with the default intensity. More precisely, we assume that $\operatorname{Corr}\left(\delta, \lambda_{d}\right)>0$. The higher the default intensity the higher the loss given default.

When pricing CDSs and LCDSs, the stochastic feature of the recovery rate has no impact on the fee leg, but on the protection leg. Using the results from Appendix A, the present value 
of the protection leg can be derived closed-form as

$$
\begin{aligned}
P V_{\text {Loss }} & =N \cdot E\left[\int_{0}^{T} D(s) \lambda_{d}(s) \exp \left(-\int_{0}^{s}\left(a \lambda_{p}(u)+\lambda_{d}(u)\right) d u+\delta(s)\right) d s\right]= \\
& =N \cdot \int_{0}^{T} D(s) \mathcal{L}_{0, s}^{\mathbf{X}}\left(\left[\begin{array}{lll}
a & 1 & 0
\end{array}\right]^{T},\left[\begin{array}{lll}
0 & 0 & 1
\end{array}\right]^{T},\left[\begin{array}{lll}
0 & 1 & 0
\end{array}\right]^{T}\right) d s
\end{aligned}
$$

where $\mathbf{X}=\left[\begin{array}{lll}\lambda_{p} & \lambda_{d} & \delta\end{array}\right]^{T}$. Concerning CDS and LCDS pricing, this means a three-factor stochastic model.

As discussed in Section 1, the recovery process is related not to the cancellation feature, but to the capital structure where the CDS or LCDS underlying points to. Obviously, a CDS and an LCDS issued on the same borrower have their own recovery processes, though we assume that these recovery processes are strongly correlated. As shown on Figure 4, it may happen, that even on the same borrower different kind of CDSs and LCDSs are issued on. These default swaps may have their own recovery processes.

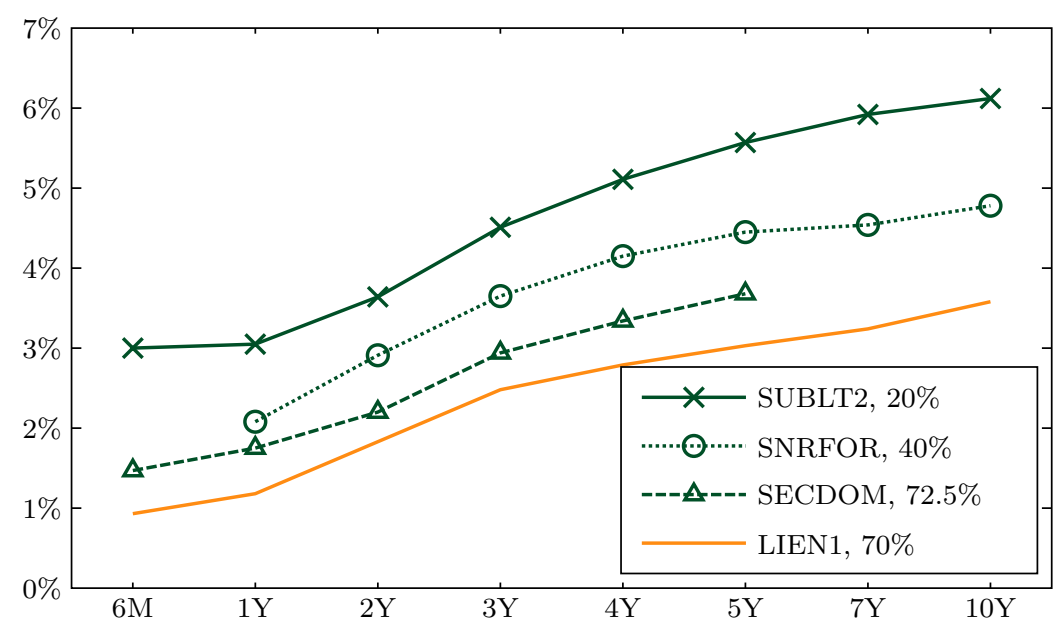

Figure 4: SUNGARD DATA Sys INC CDS (green) and LCDS (orange) curves on 31Jul08

In Markit, beside the CDS and LCDS quotes, also expected recovery rates $(\bar{R})$ are provided. On Figure 4, we indicated also these expected recovery rates. However, these rates are not traded instruments. They are only estimations and solely one estimation is given for a CDS/LCDS curve not detailed by maturities. However, this rough estimation is used when calculating the recovery-adjusted CDS spreads as discussed in Section 1.

In order to test the impact of the recovery rate as stochastic risk factor we should consider how to derive the "model" expected recovery rate. Following the market consideration, we choose to define the expected loss given default $(\bar{L})$ as the expected loss $(E L)$ weighted by the probability of default $(P D)$. Correspondingly, $\bar{R}=1-\bar{L}$.

$$
\bar{L}(\tau)=\frac{E L(\tau)}{P D(\tau)}=\frac{E\left[\int_{0}^{\tau} \lambda_{d}(s) \exp \left(-\int_{0}^{s}\left(a \lambda_{p}(u)+\lambda_{d}(u)\right) d u+\delta(s)\right) d s\right]}{E\left[\int_{0}^{\tau} \lambda_{d}(s) \exp \left(-\int_{0}^{s}\left(a \lambda_{p}(u)+\lambda_{d}(u)\right) d u\right) d s\right]}
$$

However, one may observe that the difference between the nominator above and the formula of the present value of the protection leg is only in discounting. Therefore, if we disregard from 
discounting, we may conclude that the recovery-adjusted CDS or LCDS spread is independent from the stochastic behaviour of the recovery rate and the recovery-adjustment formula from Section 1 is valid even if the recovery rate is assumed to be stochastic.

Unfortunately, this means that in case we accept the expected recovery rates given in Markit to be the expected recovery rate as specified in this section, then the market puzzle shown for instance on Figure 1 or 4 can not be explained by the multi-factor stochastic model presented in this paper.

An other puzzle related to the recovery rate is that on 31Jul08 for Ineos Gp Hldgs plc the expected recovery rate for the cancellable LCDS curve (LIEN1, EUR, CR, CANCEL, 70\%) was below the expected recovery rate for the semi-cancellable LCDS curve (LIEN1, EUR, CR, CANCEL, $72.5 \%$ ).

Intuitively, a prepayment happens likely when the market is bullish. Therefore, if the prepayment is allowed the default happens more likely when the recovery rate is lower, thus the expected loss weighted by the probability of default is lower.

In order to test whether this puzzle is explained by this model, we set up a test scenario. In this scenario the default and prepayment intensities have the same parameterization given by $\left(\lambda_{i}(0), \kappa_{i}, \theta_{i}, \sigma_{i}\right)=(4 \%, 0.5,8 \%, 2 \%)$, the loss given default process is parameterized by $(\delta(0), \kappa, \theta, \sigma)=(\ln (30 \%), 0.5, \ln (30 \%), 40 \%), \operatorname{Corr}\left(\lambda_{d}, \lambda_{p}\right)=-0.5, \operatorname{Corr}\left(\lambda_{d}, \delta\right)=0.3$ and $\operatorname{Corr}\left(\lambda_{p}, \delta\right)=-0.3$. The cancellable and the semi-cancellable LCDS curves and the corresponding expected recovery rate curves are given in Table 2.

\begin{tabular}{l|c|ccccccc} 
& & $6 \mathrm{M}$ & $1 \mathrm{Y}$ & $2 \mathrm{Y}$ & $3 \mathrm{Y}$ & $4 \mathrm{Y}$ & $5 \mathrm{Y}$ & $7 \mathrm{Y}$ \\
\hline CANCEL & $\mathrm{S}$ & $1.06 \%$ & $1.17 \%$ & $1.33 \%$ & $1.44 \%$ & $1.52 \%$ & $1.58 \%$ & $1.65 \%$ \\
$(a=1)$ & $\bar{R}$ & $68.81 \%$ & $68.18 \%$ & $67.44 \%$ & $67.06 \%$ & $66.85 \%$ & $66.72 \%$ & $66.59 \%$ \\
\hline NONCANCEL & $\mathrm{S}$ & $1.06 \%$ & $1.17 \%$ & $1.33 \%$ & $1.45 \%$ & $1.53 \%$ & $1.59 \%$ & $1.67 \%$ \\
$(a=0.1)$ & $\bar{R}$ & $68.81 \%$ & $68.18 \%$ & $67.45 \%$ & $67.07 \%$ & $66.87 \%$ & $66.75 \%$ & $66.62 \%$ \\
\hline
\end{tabular}

Table 2: Cancellable and Semi-Cancellable LCDS Curves and Corresponding Expected Recovery Rate Curves

As concluded in Section 4 in view of Figure 3, the cancellable LCDS spread curve is slightly below the corresponding non-cancellable LCDS curve. Moreover, it can be asserted that the model reproduces the market puzzle namely once both cancellable and semi-cancellable LCDSs are issued on the same borrower, the expected recovery rate is lower for the cancellable LCDS than for the semi-cancellable LCDS. (However, it should be mentioned that for some parameterization the cancellable expected recovery rate can be above the semi-cancellable expected recovery rate.)

\section{Marking-to-Market and Hedging}

In the previous sections we saw that the cancellation feature - disregarding from extreme cases - does not have significant impact on the recovery-adjusted fair spreads. One may reasonably raise the question then, why the cancellation feature is so important if it has basically no impact on the fair spread?

As we formulated our assumptions in Section 3, the cancellation probability is amortizing both the conditional default probabilities and the survival probabilities. In this manner, the cancellation probability is amortizing both the default leg and the fee leg of the LCDS. In the end, the impact of the cancellation probability on the fair spread is not significant. 
However, when using LCDSs somebody took a position on the credit market and the LCDS spreads have significantly changed in the meantime, then the cancellation probability comes into the focus and plays an important role in marking-to-market and managing the risks of the outstanding position. Due to possible cancellation the risky annuity of the LCDS, which determines basically the value of the position and used also for hedge calculations, is definitively lower than it would be when disallowing cancellation.

If both the default and cancellation intensities are constant at $5 \%$, then the risky annuity for a $5 Y$ LCDS is 4.5 years not taking the cancellation feature into account, while it is only 4 years if the cancellation feature is taken into account. This makes a huge difference in the MtM. After some tests, we may conclude that the risky annuity is highly exposed to the cancellation intensity but not to the correlation between the stochastic default and cancellation intensities.

As shown in this paper, implied cancellation probabilities can hardly be derived solely from CDS and LCDS spreads. Therefore, the pricing of outstanding LCDS positions is far underdetermined and makes the unwind cumbersome. Among others, this pricing difficulty may have contributed to the illiquidity on the LCDS markets, which put the dealers community recently to remove the cancellation feature from the LCDS contracts and propose some kind of new bullet contract as replacement.

Here, one may observe an interesting link with counterparty risk pricing in CDSs. The default of the protection seller is also a cancellation event. ${ }^{3}$ Therefore, as we concluded for prepayments we may expect that counterparty risk has no significant impact on the recoveryadjusted fair spreads, but it has an important impact on the marking-to-market. The model developed and experimented in this paper can be directly used for pricing counterparty risk in CDSs. The difference is that the sign of the correlation is likely to be the opposite.

\section{Summary}

In this paper, in order to analyse the relationship of the CDS and LCDS markets, we developed and experimented an intensity based multi-factor model, which incorporates the joint modelling of default, prepayment and recovery risks. We identified two correlations as risk factors that may explain the joint puzzle of CDS and LCDS spreads: the correlation between default and prepayment intensities and the correlation between default intensity and recovery rate.

However, as we found during the analysis, in case we accept the expected recovery rates given in Markit to be the expected recovery rate as specified in Section 5, then none of these two risk factors can explain the most important joint puzzles of the CDS and LCDS spreads. Nevertheless, the analysed two risk factors can explain some other less important market puzzles.

The results of this paper are exceedingly important for the modelling and pricing syndicated secured loan derivatives. The major difficulty when pricing these derivatives is to retrieve the implied prepayment probabilities from traded market instruments. However, as we have shown in this paper in spite of some market believes these implied prepayment probabilities can hardly be derived solely from CDS and LCDS spreads.

As far as it is known this is the first paper that analysed the CDS and LCDS markets by experimenting the explanation power of some stochastic risk factors, which was supposed earlier to explain the joint puzzle of CDS and LCDS spreads. Moreover, as far as it is known this is the first paper that provides a closed-form solution for a continuous time stochastic recovery rate model, where the recovery rate, with some reasonable parameter restriction, is kept between zero and one.

\footnotetext{
${ }^{3} \mathrm{~A}$ protection on WaMu bought from Lehman earlier should not have had a big value in the days before the collapse of Lehman.
} 


\section{Appendix}

\section{A Multivariate Gaussian OU}

Derivation. In this appendix, we set up a model based on the multivariate Gaussian OrnsteinUhlenbeck process and we derive the necessary pricing solutions in closed-form. It is supposed, that there exists an equivalent martingale measure $\mathbb{Q}$, under which the dynamics of the driving factors are given by

$$
d X_{i}(t)=\kappa_{i}\left(\theta_{i}-X_{i}(t)\right) d t+\sigma_{i} d W_{i}^{\mathbb{Q}}(t)
$$

where $\sigma_{i} d W_{i}^{\mathbb{Q}}$ stochastic components are correlated with a given covariance matrix $\Sigma$ and $\mathbf{W}^{\mathbb{Q}}$ is an $\left(\mathcal{F}_{t}\right)$-standard Brownian motion under $\mathbb{Q}$ in $\mathbb{R}^{N}$.

The multivariate Ornstein-Uhlenbeck process, described by $\mathbf{X}(t)$, is an $N$ dimensional Markov process, which is affine under the equivalent martingale measure $\mathbb{Q}$. Therefore, given $\mathbf{X}(t)$ the conditional Laplace transform of $\mathbf{X}(s)$ and its integral is an exponential-affine function of $\mathbf{X}(t)$, i.e.

$$
\mathcal{I}_{t, s}^{\mathbf{X}}(\mathbf{a}, \mathbf{z})=E_{t}^{\mathbb{Q}}\left[\exp \left(-\int_{t}^{s} \mathbf{a}^{T} \mathbf{X}(u) d u+\mathbf{z}^{T} \mathbf{X}(s)\right)\right]=\exp \left(\alpha(t, s, \mathbf{a}, \mathbf{z})+\beta(t, s, \mathbf{a}, \mathbf{z})^{T} \mathbf{X}(t)\right)
$$

where $\mathbf{a}, \mathbf{z} \in \mathbb{R}^{N}$. For notational convenience, let us rewrite the SDE of $\mathbf{X}(t)$ and emphasize the affine property of the multivariate Ornstein-Uhlenbeck process:

$$
d \mathbf{X}(t)=\left(\mathbf{K}_{0}+\mathbf{K}_{1} \mathbf{X}(t)\right) d t+\sqrt{\mathbf{H}_{0}} d \mathbf{W}^{\mathbb{Q}}(t)
$$

where $\mathbf{K}_{0} \in \mathbb{R}^{N}, \mathbf{K}_{1}, \mathbf{H}_{0} \in \mathbb{R}^{N \times N}$ and these are pure transformations of the individual $\kappa, \theta$ parameters and the common covariance matrix $\Sigma$.

As additional requirement of this paper, we differentiate the Laplace transform $\mathcal{I}$ by $\mathbf{z}$ and we note it by $\mathcal{L}$. Here, the parameter $\mathbf{m} \in \mathbb{R}^{N}$.

$$
\begin{aligned}
\mathcal{L}_{t, s}^{\mathbf{X}}(\mathbf{a}, \mathbf{z}, \mathbf{m}) & =\partial_{\mathbf{z}} \mathcal{I}_{t, s}^{\mathbf{X}}(\mathbf{a}, \mathbf{z}) \mathbf{m}=E_{t}^{\mathbb{Q}}\left[\mathbf{X}^{T}(s) \mathbf{m} \exp \left(-\int_{t}^{s} \mathbf{a}^{T} \mathbf{X}(u) d u+\mathbf{z}^{T} \mathbf{X}(s)\right)\right]= \\
& =\left(\partial_{\mathbf{z}} \alpha(t, s, \mathbf{a}, \mathbf{z}) \mathbf{m}+\mathbf{X}^{T}(t) \partial_{\mathbf{z}} \beta(t, s, \mathbf{a}, \mathbf{z}) \mathbf{m}\right) \cdot \mathcal{I}_{t, s}^{\mathbf{X}}(\mathbf{a}, \mathbf{z})
\end{aligned}
$$

Gaining from the affine property of the multivariate Ornstein-Uhlenbeck process, based on [17], $\alpha$ and $\beta$ are specified as the solution of the following two (matrix) Riccati ODEs:

$$
\begin{aligned}
& \partial_{t} \beta(t, s, \mathbf{a}, \mathbf{z})=\mathbf{a}-\mathbf{K}_{1}^{T} \beta(t, s, \mathbf{a}, \mathbf{z}) \\
& \partial_{t} \alpha(t, s, \mathbf{a}, \mathbf{z})=-\mathbf{K}_{0}^{T} \beta(t, s, \mathbf{a}, \mathbf{z})-\frac{1}{2} \beta(t, s, \mathbf{a}, \mathbf{z})^{T} \mathbf{H}_{0} \beta(t, s, \mathbf{a}, \mathbf{z})
\end{aligned}
$$

with the boundary conditions $\beta(s, s, \mathbf{a}, \mathbf{z})=\mathbf{z}$ and $\alpha(s, s, \mathbf{a}, \mathbf{z})=0$. It follows, that

$$
\beta(t, s, \mathbf{a}, \mathbf{z})=\left(\exp \left(\mathbf{K}_{1}^{T}(s-t)\right)+\mathbf{I}\right) / \mathbf{K}_{1}^{T} \cdot \mathbf{a}+\exp \left(\mathbf{K}_{1}^{T}(s-t)\right) \cdot \mathbf{z}
$$

One may recognize, that the left side of $\beta$ is related to the integral part of $\mathcal{L}$, while the right side of $\beta$ is related to the linear part of $\mathcal{L}$. This conforms with the affine property. 
Now, switching back to the original parameterization of the multivariate Ornstein-Uhlenbeck process, the solution for $\beta$ can be rewritten as

$$
\beta_{i}=e_{i} \cdot p_{i}+q_{i}
$$

where $e_{i}=\exp \left(-\kappa_{i}(s-t)\right), r_{i}=1 / \kappa_{i}, q_{i}=-a_{i} r_{i}$ and $p_{i}=z_{i}-q_{i}$. In view of these results, one may rewrite also the second ODE as follows:

$$
\partial_{t} \alpha(t, s, \mathbf{a}, \mathbf{z})=-\sum_{i=1}^{N} \kappa_{i} \theta_{i} \beta_{i}-\frac{1}{2} \sum_{i=1}^{N} \sum_{j=1}^{N} \Sigma_{i j} \beta_{i} \beta_{j}
$$

where $\Sigma_{i j}=\rho_{i j} \sigma_{i} \sigma_{j}$. The solution for this second ODE can be derived by integrating out the $\beta_{i}$ s and $\beta_{i} \beta_{j}$ s. At first, we provide these indefinite integrals:

$$
\left[\begin{array}{c}
\int \beta_{i} \\
\int \beta_{i} \beta_{j}
\end{array}\right]=\left[\begin{array}{c}
c+e_{i} p_{i} r_{i}-(s-t) q_{i} \\
c+e_{i} e_{j} p_{i} p_{j} r_{i j}+e_{i} p_{i} q_{j} r_{i}+e_{j} p_{j} q_{i} r_{j}-(s-t) q_{i} q_{j}
\end{array}\right]
$$

where $r_{i j}=1 /\left(\kappa_{i}+\kappa_{j}\right)$. Afterwards, it follows that

$$
\begin{aligned}
\alpha(t, s, \mathbf{a}, \mathbf{z}) & =c-\sum_{i=1}^{N} \kappa_{i} \theta_{i}\left(e_{i} p_{i} r_{i}-(s-t) q_{i}\right)- \\
& -\frac{1}{2} \sum_{i=1}^{N} \sum_{j=1}^{N} \Sigma_{i j}\left(e_{i} e_{j} p_{i} p_{j} r_{i j}+e_{i} p_{i} q_{j} r_{i}+e_{j} p_{j} q_{i} r_{j}-(s-t) q_{i} q_{j}\right)
\end{aligned}
$$

where satisfying the boundary condition

$$
c=\sum_{i=1}^{N} p_{i} \theta_{i}+\frac{1}{2} \sum_{i=1}^{N} \sum_{j=1}^{N} \Sigma_{i j}\left(p_{i} p_{j} r_{i j}+p_{i} q_{j} r_{i}+p_{j} q_{i} r_{j}\right) .
$$

The solution for $\alpha$ and $\beta$ fully determines the Laplace transform $\mathcal{I}$. However, to determine its derivative $\mathcal{L}$ as well, one step should be made further. It follows that

$$
\partial_{\mathbf{z}} \beta(t, s, \mathbf{a}, \mathbf{z})=\left[\begin{array}{lll}
\partial_{\mathbf{z}_{1}} \beta(t, s, \mathbf{a}, \mathbf{z}) & \cdots & \partial_{\mathbf{z}_{N}} \beta(t, s, \mathbf{a}, \mathbf{z})
\end{array}\right]=\left[\begin{array}{ccc}
e_{1} & 0 & 0 \\
0 & \ddots & 0 \\
0 & 0 & e_{N}
\end{array}\right]
$$

This derivative is basically a diagonal matrix for the multivariate Ornstein-Uhlenbeck process. Furthermore, $\alpha$ can be differentiated similarly:

$$
\partial_{\mathbf{z}} \alpha(t, s, \mathbf{a}, \mathbf{z})=\left[\begin{array}{lll}
\partial_{\mathbf{z}_{1}} \alpha(t, s, \mathbf{a}, \mathbf{z}) & \cdots & \partial_{\mathbf{z}_{N}} \alpha(t, s, \mathbf{a}, \mathbf{z})
\end{array}\right]
$$

where

$$
\partial_{\mathbf{z}_{i}} \alpha(t, s, \mathbf{a}, \mathbf{z})=\theta_{i}\left(1-e_{i}\right)+\sum_{j=1}^{N} \Sigma_{i j}\left(p_{j} r_{i j}+q_{j} r_{i}-e_{i} e_{j} p_{j} r_{i j}-e_{i} q_{j} r_{i}\right)
$$

Now, both $\mathcal{I}$ and $\mathcal{L}$ are derived in closed-form. They can be calculated quickly with few arithmetic operations. 


\section{References}

[1] Altman, E., Brady, B., Resti, A. and Sironi, A. (2005) The Link between Default and Recovery Rates: Theory, Empirical Evidence and Implications. The Journal of Business, 78(6), 2203-2228.

[2] Bakshi, G., Madan, D. and Zhang, F. (2004) Understanding the Role of Recovery in Default Risk Models: Empirical Comparisons and Implied Recovery Rates. Working Paper, Department of Finance, University of Maryland. Available at: http://www.defaultrisk.com/pp_recov_31.htm.

[3] Bartlam, M. and Artmann, A. (2007) Loan only credit default swaps - the new European standard form. Capital Markets Law Journal, 2(4), 414-426.

[4] Batterman, J., Rasmussen, I. and Yan, D. (2008) A Brief Review of "The Basis". Special Report, FitchRatings. Available at: http://www.defaultrisk.com/pp_crdrv_34.htm.

[5] Beinstein, E. and Scott, A. (2006) Credit Derivatives Handbook. Corporate Quantitative Research, JPMorgan. December, 2006.

[6] Benzschawel, T. (2007) Modeling Loan Prices, Spreads, and LCDS. The University of Chicago, Conference on Credit Risk, October 19-20, 2007. Available at: http://stevanovichcenter.uchicago.edu/conferences/creditrisk/Benzschawel.ppt.

[7] Berndt, A., Jarrow, R.A. and Kang, C. (2007) Restructuring Risk in Credit Default Swaps: An Empirical Analysis. Stochastic Processes and their Applications, 117(11), 1724-1749.

[8] Brennan, M. and Schwartz, E. (1980) Analyzing Convertible Bonds. Journal of Financial and Quantitative Analysis, 15(4), 907-929.

[9] Cheng, P. and Scailett, O. (2005) Linear-quadratic jump-diffusion modelling with application to stochastic volatility. Working Paper, HEC Geneva.

[10] Christensen, J.H.E. (2005) Joint Estimation of Default and Recovery Risk: A Simulation Study. Working Paper, Department of Finance, Copenhagen Business School. Available at: http://www.defaultrisk.com/pp_recov_81.htm.

[11] Christensen, J.H.E. (2007) Joint Default and Recovery Risk Estimation: An Application to CDS Data. Working Paper. Available at: http://www.defaultrisk.com/pp_crdrv_37.htm.

[12] Coffey, M. (2007) LCDS and Loan Spreads. LPC Gold Sheets, 21(11) 1-24. Reuters Loan Pricing Corporation Publication.

[13] Cox, J., Ingersoll, J. and Ross, S. (1985) A Theory of the Term Structure of Interest Rates. Econometrica, 53(2), 385-407.

[14] Dobránszky, P. and Schoutens, W. (2008) Generic Lévy One-Factor Models for the Joint Modelling of Prepayment and Default: Modelling LCDX. Technical Report 08-03, Section of Statistics, K.U. Leuven. Available at: http://wis.kuleuven.be/stat/Papers/TR0803.pdf.

[15] Duffie, D. (1998) First-to-Default Valuation. Working paper, Graduate School of Business, Stanford University. Available at: http://www.stanford.edu/ duffie/first.ps.

[16] Duffie, D. (2005) Credit risk modeling with affine processes. Journal of Banking and Finance, 29(11), 2751-2802. 
[17] Duffie, D., Pan, J. and Singleton, K. (2000) Transform analysis and asset pricing for affine jump-diffusions. Econometrica, 68(6), 1343-1376.

[18] Elizalde, A. (2007) Pricing cancellable LCDS. Credit Derivatives Strategy, Merrill Lynch. February, 2007.

[19] Gourieroux, C. and Sufana, R. (2003) Wishart Quadratic Term Structure Models. Working Paper, University of Toronto.

[20] Heston, S.L. (1993) A Closed-Form Solution for Options with Stochastic Volatility with Application to Bond and Currency Options, The Review of Financial Studies 6(2), 327343.

[21] Hull, J. and White, A. (1990) Pricing interest-rate derivative securities. The Review of Financial Studies, 3(4), 573-592.

[22] Karoui, L. (2007) Modeling Defaultable Securities with Recovery Risk. Working Paper, McGill University. Available at: http://www.defaultrisk.com/pp_model131.htm.

[23] Morgan, S. and Zheng, Z. (2007) From CDS to LCDS: Accounting for Cancellation. Quantative Credit Research Quarterly. Lehman Brothers. Vol. 2007-Q3/4.

[24] Price, T., Casey, O. and Huscher, D. (2007) Loan CDS Report. Markit. April, 2007.

[25] Toft, K. and Reyfman, A. (2003) What is the real value of restructuring? Risk Magazine, 16(6).

[26] Vasicek, O. (1977) An Equilibrium Characterisation of the Term Structure. Journal of Financial Economics, 5(2), 177-188.

[27] Wei, Z. (2007) Valuation of Loan CDS Under Intensity Based Model. Working Paper, Department of Statistics, Stanford University. Available at: www.stanford.edu/ zhenwei/papers/lcds.pdf. 